\title{
Civilisations
}

Revue internationale d'anthropologie et de sciences

humaines

43-2 | 1996

Problèmes africains contemporains

\section{Le cas du Niger : les touareg du passé au futur}

\section{Maman Saley}

\section{OpenEdition}

\section{Journals}

Édition électronique

URL : http://journals.openedition.org/civilisations/1568

DOI : 10.4000/civilisations. 1568

ISSN : 2032-0442

\section{Éditeur}

Institut de sociologie de l'Université Libre de Bruxelles

\section{Édition imprimée}

Date de publication : 1 avril 1996

Pagination : 65-82

ISBN : 2-87263-160-7

ISSN : 0009-8140

\section{Référence électronique}

Maman Saley, «Le cas du Niger : les touareg du passé au futur », Civilisations [En ligne], 43-2 | 1996, mis en ligne le 29 juin 2009, consulté le 19 avril 2019. URL : http://journals.openedition.org/ civilisations/1568; DOI : 10.4000/civilisations. 1568

\section{(c) Tous droits réservés}




\title{
Le cas du Niger: les touareg du passé au futur.
}

\author{
Maman SALEY \\ Université de Niamey
}

\section{Summary}

The objective is to put into light certain historical and cultural aspects of the Targuie civilization and to establish a ìdetailedî point of view regarding a certain number of problems that the young Nigerian democracy is confronted with. He points out that many false rumors concerning the Tuareg are diffused in the West. After a short historical brief on the Tuareg (Targui origin and population on Nigerian territory), the author describes the Tuaregís socio-political structures and the Targuieís economy. He wonders what future awaits the Sahel people. According to him, interrelations between stockbreeders and farmers constitute a system adapted to the social, economic and cultural situation of the Sahel people which favours the exploitation of natural ressources without putting the equilibrum of the sub-regionis ecosystem in danger. Therefore, Nigerians should get together in order to mould their destiny and hence apply the principles of human rights based on equality, justice and union of different ethnic groups.

Nul n'ignore aujourd'hui que l'Afrique va mal. Les causes de cet état de fait sont multiples et complexes: colonisation, 
démographie galopante, calamités naturelles, guerres, gestion népotique et désastreuse des affaires de l'État...

C'est dans cet état de fait même, que dans de nombreux pays africains, se pose actuellement le vaste problème de fonder idéologiquement et réaliser dans les faits une organisation politique nationale qui prenne chacun en compte. La marge de manoeuvre est étroite, les moyens sont limités, le défi est immense. Dans de telles situations, il est très important de fonder les mesures politiques qui sont prises sur des valeurs clairement exprimées et acceptées par tous après avoir été mises sur la table du débat.

Il est aussi essentiel de faire usage des outils conceptuels et de façons de faire locales. Il y a là tout un savoir vivre, un savoir penser et un savoir faire que les regards tournés vers l'Occident ont souvent trop dévalorisés.

Les sciences sociales, dans notre cas, l'histoire, peuvent refaire une juste place à ces traditions.

Voilà le cadre dans lequel nous souhaitons intégrer la présente étude. Son objet est circonscrit. Il s'agit de mettre en relief certains aspects historiques et culturels de la civilisation targuie et d'esquisser ainsi une vue "en profondeur" par rapport à quelques unes de questions qui se posent à la jeune démocratie nigérienne.

La perception que l'on a à l'étranger, en Europe particulièrement, de la situation politique actuelle des Touareg et de leur rapport aux structures politiques nationales est donc largement biaisée.

Beaucoup de fausses vérités ont été véhiculées, par ignorance des réalités ou par intérêt. En France, on a pu lire ou entendre ces dernières années des affirmations telles que " De race blanche, les Touareg des régions septentrionales du pays supportent très mal, comme au Mali, la tutelle d'un 
gouvernement exclusivement composé de Noirs"l, ou "Voyant leur communauté (...) négligée par les autorités, mal desservie par les organismes internationaux chargés de leur apporter une aide, ils ont engagé un combat"2 ou encore "Le peuple Touareg est menacé d'extinction"?.

Ces affirmations cachent bien des réalités historiques, pourtant nécessaires, pour une bonne compréhension de la question touarègue contemporaine. Ces réalités sont davantage masquées encore lorsque des opportunistes de tout bord saisissent l'occasion pour faire ingurgiter, avec une forte dose de romantisme et d'exotisme, à l'opinion internationale, l'idée qu'il y a une "tragédie" touarègue.

A travers cet article, nous ne prétendons pas apporter une réponse définitive à la question, mais simplement évoquer les faits historiques, car nous sommes convaincus que le passé aide à comprendre le présent et à mieux construire l'avenir.

Nous proposons donc de porter un regard rétrospectif sur les Touareg, leurs façons de vivre et leurs rapports avec d'autres populations. L'article se terminera sur le questionnement d'un document politique contemporain rédigé par des Touareg.

\section{Bref aperçu historique sur les Touareg.}

Les Touareg sont des peuples nomades qui vivent de nos jours dans le Sahara central et méridional. Ils sont estimés à plus

\footnotetext{
I MARTIN FRANÇOIS: Le Niger du Président Diori Hamani, éd L'Harmattan, Paris, 1991, p 106.

2 SUBTIL M.P.: in le Monde du 12 Février 1992, P 4.

3 EDGAR PISANI a fait cette déclaration au cours d'une interview accordée à la TVS Europe en février 1992.
} 
de trois millions ${ }^{4}$ d'habitants répartis en Algérie, au Burkirna Faso, en Libye, au Mali, en Mauritanie et au Niger.

Depuis leur mise en place dans la sous région, ils vivent de leur activité pastorale, du commerce transsaharien et, dans une moindre mesure, des produits de leur artisanat.

La littérature occidentale utilise le terme Berbère pour désigner non seulement l'ensemble de la population blanche de l'Afrique septentrionale, mais aussi celle qui vit au Burkina Faso, au Mali et au Niger.

Cette appellation se réfère surtout, aux dires de D.Hamani, à la parenté linguistique de ces peuples. Cependant la population en question utilise le terme Touareg pour se désigner. Aujourd'hui, sont appelés Touareg, les Berbères parlant le Tamashek, portant dans certains cas le voile "Taguelmust" et utilisant le Tifinagh comme écriture.

On ajoute à ce groupe les anciens captifs des Touareg (nommés Bella ou Buzu) ${ }^{5}$ et les métis issus de l'union entre eux et les Touareg.

\section{L'origine des Touareg.}

D.Hamani , historien spécialiste de la question targuie, donne plusieurs versions sur l'origine du terme Touareg et donc sur l'origine du peuple touareg. Nous proposons quelques unes de ces versions selon lesquelles le terme Touareg proviendrait de la racine "Taraka" qui, en arabe, signifie abandon.

4 source AFP, 22 octobre 199]

5 BELLA, BUZU: ces termes sont respectivement utilisés par les Zarma et les Hausa. 
Dans une première version D.Hamani ${ }^{6}$ rapporte que douze fois, les Berbères adoptèrent l'Islam. A la suite de ces apostasies ils auraient été appelés "Tawarik"; c'est-à-dire ceux qui ont abandonné l'Islam.

Dans une autre version, toujours liée à la racine Taraka, les Touareg seraient issus d'un métissage entre Berbères et Arabes. Selon cette version, un Arabe du nom de Diap al Halali tua un jour un démon(Afrit) près d'un village berbère. Le monstre exigeait chaque jour une jeune fille vierge. Lorsque les villageois demandèrent à l'Arabe ce qu'il voulait comme récompense, celui-ci, ayant avec lui 40 compagnons, demanda 40 jeunes filles vierges pour ses hommes. Les femmes restèrent seules après le départ de Diab et ses compagnons. Ainsi, celles qui étaient grosses et qui accouchèrent après le départ de leurs maris appelèrent leurs enfants Touarek, c'est-à-dire abandonnés.

Il est à noter que ces deux versions renvoient aux rapports entre les Touareg et d'autres populations ou cultures. Les rapports sont marqués la rupture. Plus encore, c'est dans la rupture que l'identité targuie plonge ses racines selon ces versions.

Selon une troisième version, le mot Touareg serait une transformation du mot Targa, lequel désignait une vallée du Fezzan (région du sud-ouest de la Libye).

Il semble que les Touareg appellent le Fezzan Targa, et les Arabes qui entrèrent en contact avec les Berbères nomades de cette région les appelèrent Touareg. Cela parce que eux-mêmes (les Berbères) se dénommaient Kel Targa (gens de Targa). Progressivement le nom Touareg se serait étendu à l'ensemble des Berbères de la sous-région.

6 HAMANI D.: op, cit, P 53. 


\section{Peuplement targui dans l'espace nigérien.}

Au Niger les Touareg sont estimés de nos jours à 700.000 habitants $^{8}$. Ils sont surtout répartis dans le centre et le nord du pays.

A l'origine ils peuplent l'Aïr et ses pourtours. Ils nomadisent au rythme des saisons des pourtours de l'Aïr jusqu'au sud du pays, c'est-à-dire dans la zone des cultures sous pluies, de la latitude $14^{\circ}$ nord à la latitude $22^{\circ}$ nord.

Les Touareg qui peuplent l'Aïr et ses environs portent le nom de Kel Ayar(ceux de l'Aïr). Parmi les principales tribus qui composent ce groupe on compte les Kel Away, les Kel Ferwan, les Kel Fade, les Ikaskazan, les Kel Tadale, les Inussafa, etc. A ces tribus on peut ajouter les Itesayan, les Kel Geres, les Illisawan, les Imuzarag, etc.

Certains de ces groupes se sont très tôt détachés du groupe Kel Ayar. Il s'agit des Ibarkoreyan, des Iwellimeden, des Ijadaranin.

L'arrivée des premiers Touareg dans la région remonterait au VIIIe siècle ${ }^{9}$. Dès le début du IXe siècle, certaines tribus étaient proches de l'Aïr d'après Hamani. Le groupe le plus ancien serait, selon toujours la même source, celui des Sandale, suivi de celui des Kel Gerès puis les Kel Away.

Ces groupes ont trouvé le massif de l'Aïr et ses environs largement occupés par une population noire parlant la langue hausa. Toutes les sources historiques sérieuses attestent la primauté de la présence hausa dans la sous-région. L'explorateur allemand Barth, qui a visité le pays entre 1850 et 1855 , nous rapporte que" le nom Air, que les Arabes orthographient Ahir, est cité pour la première fois par Léon l'Africain, dans son ouvrage

8 Recensement général de la population, Ministère du plan, Niamey, 1988.

9 HAMANI D. , op, cit, P 85. 
écrit en 1526. Il paraît donc ne pas être le nom primitif de la contrée mais provenir plutôt des conquérants berbères ou Masigh. Celui que portait antérieurement le pays, devait être Asben ou Absen, qui est encore en usage parmi les nègres et les diverses peuplades qui y demeurent"10.

Nicolaisen aussi est de cet avis lorsqu'il écrit que " Mes recherches confirmèrent pleinement ces affirmations. Tous les Touareg interrogés à propos de l'histoire de l'Aïr semblent admettre que les agriculteurs de race noire vivaient dans la régions avant eux"11

En ce qui concerne les Hausa nous avons pu constater qu'ils ne connaissent pas le terme Aïr. Ils désignent le massif et ses environs par le terme Abzin(Absen) qui est le pays de leur origine $^{12}$

Dans les pourtours sud de l'Aïr les Hausa ont fondé de nombreuses villes. Maranda était la plus importante en $650^{13}$. Sous la pression écologique d'une part et, l'arrivée des Touareg, eux-mêmes bousculés par l'invasion hilalienne de l'autre, une partie de la population autochtone entreprit un mouvement de migration vers le sud.

Cependant la seconde partie de ces Hausa resta sur place et constitue encore le fond de la population noire de cette région. Ce n'est pas un hasard, si de nos jours encore, il existe de nombreux termes hausa dans le parler tamashek de cette région.

10 BARTH H: Voyages et découvertes en Afrique septentrionale et centrale, éd $\mathrm{Fr}$ Van Mecnen et Cie, Bruxelles, 1860,Tome I, P202.

11 NICOLAISEN J: Structures politiques et sociales des Touareg de l'Air et de l'Ahagar, Etudes Nigériennes, N7, Niamey, 1962, P 15.

12 Pour en savoir davantage voir SALEY M in Les Etats tsotsebaki des origines au XIXe siècle, thèse de doctorat, U.L.B., 1994.

13 HAMANI D : op, cit, P 125. 


\section{La civilisation targuie.}

La civilisation targuie est avant tout celle de nomadisme. Cependant, le commerce transsaharien et la pratique quasi permanente des rezzous, dont l'origine remonte à l'arrivée des Touareg dans la sous région, occupe une place non négligeable dans le mode de vie des Touareg.

Ces dernières années, les modifications climatiques, plus que les pressions démographiques, ont imposé une nette tendance vers une sédentarisation de ce peuple. Cette situation n'est pas sans conséquence sur les structures politiques et sociales des Touareg.

\section{Les structures socio-politiques des Touareg.}

La société targuie est une société matrilinéaire fortement hiérarchisée qui s'organise en confédération, laquelle regroupe un ensemble de tribus au sein desquelles le campement reste l'unité de base.

Soulignons que l'histoire politique des Touareg est dominée par des luttes intestines fréquentes entre tribus. En dehors des Iwillemmeden qui restèrent relativement à l'écart, il y eut de violents accrochages, particulièrement entre les tribus des Kel Geres et des Itesayan ${ }^{14}$ d'une part et les Kel Away, d'autre part. Ces conflits aboutirent à l'expulsion des Kel Geres et des Itesayan de l'Aïr. ${ }^{15}$

La confédération ou Ettebel ${ }^{16}$ rassemble des tribus selon les circonstances politiques ou guerrières. Il semble qu'il existe deux principales confédérations: celle des Sandale, les plus

14 HAMANI.D, op, cit P 313 et 316.

15 HAMANI.D, op, cit P 399.

16 MALAM ISSA M. op, cit P 45. 
anciens dans le territoire dont le chef prenait le titre d'Amanokal, et celle des Kel Away, le chef porte le titre d'Anastafidet.

L'Amanokal tout comme l'Anastafidet jouissent d'une sorte de délégation de pouvoir pour défendre les intérêts des classes aristocratiques Sandal ou Kel Away. Soulignons que ces chefs n'exercent qu'un pouvoir précaire sur les diverses tribus qu'ils représentent, car ils peuvent être relevés de leur fonction par leurs électeurs.

C'est au sein de ces tribus qu'on retrouve la structure pyramidale de la société targuie. On distingue ainsi la couche des privilégiés comprenant des Imajeghan (aristocratie guerrière) des Imghad (des vassaux) et des Inesleman (marabouts). Puis vient la couche des défavorisés dont les Inadan (les artisans), les Iderfan (les affranchis) et les Iklan (les serviteurs).

\section{L'économie targuie.}

Le Sahara, cet immense océan d'erg et de reg, n'a pas toujours été perçu comme une calamité naturelle mais plutôt comme une ressource économique. En effet, pendant que les noirs d'Afrique occidentale tournaient le dos à l'océan Atlantique, leurs regards étaient tournés vers le désert d'où ils importaient et exportaient leurs marchandises vers le Maghreb et le monde méditerranéen.

Durant toute la période précoloniale, particulièrement du début de notre ère au XIXe siècle, le chameau a été le seul trait d'union entre le monde maghrébin et l'Afrique Occidentale.

Le commerce transsaharien, drainant l'essentiel des marchandises entre ces deux mondes, a favorisé l'expansion de nombreuses villes (entre autres, Agadès, Tahoua, Zinder au Niger, Tombouctou et Gao au Mali). Les empires du KanemBornu, du Mali, du Songhai, les États Hausa et tout dernièrement le Damagaram, doivent leur période de grandeur à ce commerce 
transsaharien mené par les Touareg. Durant cette période florissante qui a duré plus de 1000 ans, dans l'espace nigérien, les Touareg menaient de front, entre autres activités, l'élevage et le commerce transsaharien.

Mais l'activité principale des Touareg est l'élevage des camélidés. Ceux-ci leurs fournissent aujourd'hui encore lait et moyen de transport. Les Touareg élevaient également des ovins, des caprins et occasionnellement des bovins.

Cet élevage est pratiqué à travers un système économique communément appelé le nomadisme. Planhol et Rognon le décrivent d'ailleurs en ces termes: "Le nomadisme pastoral, exploitation du sol par l'intermédiaire du bétail, fondé sur une vie errante à la recherche du pâturage, a été le seul genre de vie assurant une mise en valeur totale et continue du désert, au- delà de la limite de la culture pluviale et entre les foyers sporadiques de l'agriculture irriguée." 17

Ce nomadisme pastoral, avec la détérioration climatique de ces dernières années, a connu de profondes mutations, qui ont d'ailleurs accru davantage l'interdépendance entre éleveurs et agriculteurs.

Pour pallier l'insuffisance du pâturage en dessous de l'isohyète $300^{18}$ les nomades, Peuls et Touareg compris, pratiquent la transhumance ponctuée par la "cure salée" et le "Hotungo"19.

17 PLANHOL et ROGNON cité par MONOD Th. in Pastoralism in tropical Africa, Oxford University Press, 1975, P 9.

18 L'isohyète 300 constitue la limite nord de culture sous pluie au Sahel. En effet le millet est une céréale sobre qui peut se cultiver avec un minimun de $300 \mathrm{~mm}$ de pluie par an.

19 La cure salée et le Hotungo: Pour la cure salée chez les Touareg les animaux effectuent un déplacement de 400 à $500 \mathrm{~km}$ en moyenne pour se regrouper dans le sud ouest d'Agadès notamment dans la région d'In Gall où les sols et puits sont naturellement salés. Chez les Peuls, Hotungo signifie le retour. 
La transhumance est une pratique pastorale qui, au-delà de sa fonction première qui consiste à faire pâturer le bétail d'une zone à l'autre au rythme des saisons, constitue un cadre idéal de relations et d'échanges entre nomades et sédentaires.

Ainsi à la fin de chaque saison hivernale et après que les paysans sédentaires aient engrangé leur récoltes, les éleveurs nomades descendent vers le sud agricole pour accéder à la vaine pâture. A cette occasion des arrangements ont lieu entres éleveurs et agriculteurs dont le principe est le suivant: l'éleveur fournit fumure, lait, et autres produits d'élevage contre des céréales et des services de l'agriculteur.

Au fur et à mesure que la saison sèche s'achève et que l'hivernage s'annonce, les nomades fuyant les pluies intenses du sud, souvent malsaines pour le bétail, remontent progressivement vers le nord.

Cette remontée se termine autour de points précis où les nomades procèdent à un rassemblement annuel, devenu quasi rituel. Ce rassemblement est généralement connu sous le nom de cure salée chez les Touareg alors que les Peuls l'appellent Hotungo.

La cure salée tout comme le Hotungo sont extrêmement importants chez ces peuples nomades. Au-delà du rassemblement du bétail qui a l'occasion de brouter un pâturage frais, ils sont l'occasion d'échanges sociaux intenses entre les nomades.

Chez les Peuls par exemple, le Hotungo favorise de grandes retrouvailles au cours desquelles certains rites (baptêmes, mariages), et les résolutions des différends se déroulent. Les éleveurs restent sur les lieux de ces grands rassemblements jusqu'à la fin de la saison des pluies et le cycle recommence.

Le commerce transsaharien a beaucoup contribué à l'enrichissement non seulement de l'aristocratie targuie qui 
prélevait des taxes sur les produits qui transitèrent par son territoire, mais aussi de certaines couches des Imajeghan qui participèrent au trafic en assurant le transport des marchandises entre le Maghreb et les villes des États hausa.

Ce commerce portait tant sur des produits agricoles(les céréales, les peaux, le beurre, l'indigo, le henné, etc.) que sur des marchandises telles les esclaves, le sel les plumes d'autruche, les textiles, les armes, etc.

Les conséquences économiques et sociales de ce commerce sont multiples et séculaires dans la sous région

Du point de vue économique, Agadès, Tahoua, Tanout et Zinder ont connu leur période de grandeur pendant cette époque du commerce transsaharien. S'agissant de Zinder, Baiers écrivait que "Zinder was not only a stopping place for trans-saharan caravans, but also the point of contact between the pastoral and sedentary economies at the edge of desert. The exchange of animals, millets, salt, cloth, and potash took place on a long scale, and the boom conditions the trans-saharan trade stimulated the growth of this internal trading setwork." 20

Cette expansion économique fut suivie d'un peuplement targui vers le sud. A Zinder, par exemple, les Kel Away seraient la première fraction targuie à s'installer en $1822^{21}$ sous le règne du sultan Ibram. Ils seraient à l'origine de la création du quartier Zango $^{22}$ de Zinder.

20 BAIERS S. An economic history of central Niger, Clarendon Pres. Oxford 1980, P 2

\section{BAIERS S: op, cit, P 19}

22 Zango: mot hausa désignant à l'origine une palissade à caractère provisoire entourant un campement. Par la suite les Hausa utilisent ce terme pour désigner l'habitat ou un quartier d'une population étrangère. Ce terme indique de nos jours un habitat ou quartier des Hausa hors de leur territoire ou à l'étranger. 
L'agriculture est rarement pratiquée autour des points d'eau et dans les oasis où les Touareg cultivent du blé, des dattes, du poivron, de l'ail, etc.

Dans les zones des cultures de sous-pluie, l'agriculture n'est pas pratiquée par les Touareg mais plutôt par leur captifs, notamment les Iklan. Toutefois la production agricole est loin d'être suffisante. Elle ne constitue qu'un appoint que les Touareg doivent compléter à travers des échanges ou par des rezzous sur les populations sédentaires.

Pendant toute la période précoloniale, l'esclavage était largement pratiqué et les esclaves, dont le trafic a été hautement lucratif, constituaient une source économique substantielle dans toute la sous région. Il y a lieu de rappeler que l'esclavage n'est pas l'apanage des seuls Touareg.

Bien avant eux, d'autres peuples Kanuri, Hausa, Zarma, Peul) l'avaient pratiqué dans des proportions beaucoup plus importantes d'ailleurs. A titre d'exemple, dans les milieux aisés des États hausa, on comptait, jusqu'en 1898, 60 à 100 esclaves $^{23}$ par famille. Même lors d'un mariage, la jeune mariée était accompagnée à son domicile conjugal par une ou deux esclaves. L'origine de ces pratiques est aussi vieille que les structures politiques de ces peuples ${ }^{24}$.

Voici esquissé très brièvement, le tableau politique, économique et social avant la présence européenne. Les circonstances historiques intervenues au début de ce siècle (la colonisation) et surtout les dégradations des conditions climatiques de ces dernières années, ont porté un coup décisif à toute cette civilisation. Elles ont profondément modifié la configuration socio-économique non seulement des nomades, mais de tous les peuples de la sous région.

23 SMITH M.: Baba de Karo, éd Plon, Paris , 1969, P 14

24 Pour en savoir plus voir SALEY M. op, cit, P 194. 


\section{Problèmes africains contemporains}

Depuis la mise en place du système colonial, le commerce trans-saharien a été totalement détourné au profit des régions côtières et, d'autre part, l'usage du véhicule à moteur a fortement concurrencé le chameau, entraînant ainsi une perte considérable des revenus des Touareg.

Par ailleurs, les rezzous, l'esclavage et le commerce qu'ils entraînent qui constituaient une source de revenu non négligeable pour les Touareg, ont été interdits par la colonisation. D'autre part, à la faveur du tourisme, les Touareg ont développé la fabrication et la vente d'objets artisanaux.

\section{Quel avenir pour les peuples du Sahel?}

Les problèmes que vivent les Touareg pour lesquels les rebelles ont ouvert les hostilités ne sont pas spécifiques à ce peuple encore moins aux seuls nomades. C'est tout le Sahel qui est concerné. Personne ne peut contester aujourd'hui que tous les indicateurs économiques et sociaux de ces pays sont au rouge.

L'avenir est dans l'union autour de la terre. Nous pensons que la revendication d'un territoire par telle ou telle autre ethnie ou peuple doit être dépassée pour faire place à une cause plus noble que des considérations ethnocentriques et égoïstes. Ce serait conforme à la grande philosophie des Africains qui, on le sait, ont une conception du bien communautaire aussi ancienne que leur origine.

Pierre Salmon écrit non sans raison que chez l'Africain "le principe de la propriété communautaire prime sur celui de la propriété privée"25. Dans la philosophie africaine, des éléments comme la terre, l'eau, l'air, le pâturage sont inaliénables mais appartiennent à toute la communauté. Chaque membre de la

25 SALMON P., L'Afrique meurt, sommes -nous concermés?, éd Synedi/RTBF,1994, p104. 
communauté y a droit. De même au Niger, les Touareg ont droit à la terre, à l'espace mais pas plus que d'autres ethnies ou peuples.

De nos jours, l'identité targuie est avant tout l'appartenance à une communauté linguistique, mais c'est aussi la pratique du nomadisme. Or le nomadisme tel que nous l'avons évoqué, c'est un mode économique basé sur la transhumance d'une zone à une à autre, d'une région à une autre, se nourrissant de l'interdépendance entre éleveurs et agriculteurs.

Cette interdépendance nous convainc qu'aucune société nomade ne peut subsister sans contact avec une société sédentaire, car l'échange qui en découle est d'une importance capitale entre les deux communautés. C'est pourquoi nous pensons que la transhumance, une des manifestations du nomadisme, est un système économique adapté à nos réalités sociales, économiques et culturelles et qui permet une meilleure exploitation de nos ressources naturelles sans compromettre l'équilibre de l'écosystème de la sous région.

Il serait absurde, en plus des barrières fixées par la colonisation et qui ont servi de frontières à nos États, de créer d'autres limites au pastoralisme. On ne peut prétendre défendre le nomadisme en le confinant dans des réserves elles-mêmes en proie à la désertification.

On ne peut pas ne pas marquer son étonnement lorsque des Touareg, parlent de la marginalisation de leur communauté. Si au Niger il y a une ethnie qui, depuis l'accession du pays à l'indépendance, a une meilleure représentation que les autres, proportionnellement à son nombre, au sein des gouvernements qui se sont succédés, c'est bien celle des Touareg.

A. Salifou précise même que dans chacun des trente huit gouvernements qui se sont succédés au Niger, il eut deux ministres touareg en moyenne, ce qui leur donne une 


\section{Problèmes africains contemporains}

représentation de $22 \%$ de l'équipe gouvernementale pour une population qui ne représentait que 8 à $9 \%{ }^{26}$, de celle du pays.

Selon la même étude, les Hausa, de loin l'ethnie majoritaire avec plus de $50 \%$ ne sont représentés en moyenne que par quatre ministres par gouvernement. Nous nous passerons des détails de la participation des cadres touareg au sein de l'armée, de l'administration et cadres diplomatiques, participation que Salifou a largement évoquée dans son livre la question touareg au Niger.

\section{Les droits de l'homme, le Niger et les Touareg.}

Il n'existe aujourd'hui sur terre, aucun État, aucune organisation politique sérieuse qui ne manifeste son souci de réaliser les droits de l'homme, affirmés sur le plan international le 10 décembre 1948 par l'Assemblée générale des Nations Unies. Tous les États, toutes les organisations politiques s'en inspirent pour édifier leur politique. Même l'Afrique du sud qui les a longtemps bafoués a rejoint le rang des Nations civilisées.

Rappelons que cette déclaration universelle des droits de l'homme consacre essentiellement l'égalité entre les hommes, le droit politique, et le droit à la citoyenneté. Des Touareg ont rédigé une plaquette intitulée: Programme cadre de la rébellion. Dans ce document ils se posent en rebelles et lancent bon nombre de revendications qui sont de toute évidence en rupture avec la philosophie africaine.

Il s'agit de revendications non seulement à caractère autonomiste mais aussi qui légitiment l'inégalité entre les hommes puisque le titre II, chapitre 7 de leur programme politique indique:"Seuls les populations Touarègues, Arabes, Kawariennes et les Peuls Bororo autochtones sont électrices et éligibles. Les résidents venus des autres régions du pays pour

26 SALIFOU A: La question touareg au Niger, éd Karthala, 1993, P85. 
des raisons commerciales, administratives et autres ne sont ni électeurs ni éligibles."

Il vaudrait mieux appeler au débat philosophique sur des valeurs fondatrices (Nations Unies) et la façon de les mettre en oeuvre dans l'organisation de l'État.

\section{Conclusion}

Nous connaissons aujourd'hui un désastre économique. Les détériorations des conditions climatiques intervenues ces dernières années ne sont pas pour arranger les choses. A présent, c'est ensemble que les Nigériens doivent faire face à leur destin., pour combattre la misère, le désert et les autres fléaux. La kalachnikov ou tout autre arsenal n'est certainement pas la meilleure arme pour un tel combat. 


\section{Bibliographie}

-A.F.P.... du 22-10-1991

-BAIERS S: An economic history of central Niger, Clarendon Press, Oxford 1980. -BARTH H : Voyages et découvertes en Afrique septentrionale et centrale, éd Fr Van Meenen et Cie, Bruxelles, 1860, Tome I.

-BOURGEOT.A: Politique Africaine $N^{\circ} 48$. "Le Niger, Chronique d'un Etat", éd Karthala, 1990.

-EKADE GHALIO: Problème méthodologique de l'étude démographique des nomades, Mémoire de maîtrise, U.C.L, 1992.

-Encyclopédie Universalis: Thésaurus Index, Malesherbes, 1991.

-FRANÇOIS MARTIN: Le Niger du Président Diori Hamani, éd L'Harmattan, 1991, PP 420.

-HAMANI.D: "Sultanat Touareg de l'Ayar", dans Etudes Nigériennes, Nº5, Niamey, 1989.

-GALATY: J.G. L'avenir des peuples pasteurs, Université Mc Gill, Montréal, 1983, PP 432.

-CLAUDOT-HAWAD H.: Touareg. Exil et Résistance, Edisud, 1990, PP198.

-MALAM ISSA MAHAMAN : Le Damergu du XVIe au XIXe siècle: repeuplement et formation de l'Etat targui des Immuzurag, Université de Côte d'Ivoire, 1990.

-MANO DAYAK: Touareg, la tragédie, éd Lattès, 1993, PP 217.

-SMITH M.: Baba de Karo, éd Plon , Paris 1969.

-NICOLAISEN J: "Structures politiques et sociales des Touareg de l'Aïr et de l'Ahagar", dans Etudes nigériennes, n7, Niamey, 1962.

-SALMON P.: L'Afrique meurt, sommes-nous concernés? éd Synedi/R.T.B.F, 1994.

-SALIFOU.A: L'histoire du Niger, Unesco-ACCT.

-SALIFOU A : La question touarègue au Niger, éd Karthala, Paris, 1993, PP 207.

-SALEY MAMAN : Les Etats tsotsebaki des origines au XIXe siècle, thèse de doctorat, U.L.B., 1994, PP 520.

MONOD Th.: Pastoralism in tropical Africa, Oxford University Press, 1975 